\title{
Selective Synthesis of Fluorinated Compounds: Process Development and Preparation of Fluorinated Compounds from Gram up to Kilogram Scale
}

\author{
Heinz Steiner* and Hans Meier
}

\begin{abstract}
The most versatile fluorination reactions for the preparation of up to $\mathrm{kg}$ amounts of fluorinated compounds are presented, i.e. the deoxofluorination of alcohols, oxo compounds and carboxylic acids using $\mathrm{SF}_{4}$, and the conversion of anilines to fluoro aromatics. Furthermore the enantioselective catalytic fluorination of $\beta$-ketoesters is highlighted. The option of outsourcing preparative fluorination is discussed.
\end{abstract}

Keywords: Benzotrifluorides · Deoxofluorination · Fluorination · Fluoroaromatics · Schiemann reaction

\section{Introduction}

Fluorinated compounds are often used in research laboratories of the pharma and crop protection industry. This is due to the fact that fluorine atoms often enhance the desired properties of biologically active compounds. Today, research and development chemists can purchase many commercially available fluorinated molecules as building blocks for a fluorinated target molecule. If this is not the case, a plethora of methods exist to selectively fluorinate a variety of substrate classes [1]. In this contribution we would like to discuss two aspects: 1) The scope of selected fluorination methodologies and 2) the option of outsourcing the preparation of up to $\mathrm{kg}$ quanti-

${ }^{\star}$ Correspondence: H. Steiner

Solvias AG

P.O. Box

$\mathrm{CH}-4002$ Basel

Tel.: + 41616866305

Fax: + 41616866311

E-Mail: heinz.steiner@solvias.com ties of fluorinated intermediates to a specialized contractor such as Solvias.

\section{Useful Fluorination Methods}

Several fluorination methods allow the selective formation of carbon-fluorine bonds in organic molecules on a preparative scale:

- Schiemann reaction

- Deoxofluorination using $\mathrm{SF}_{4}$

- Electrophilic fluorination using $\mathrm{F}^{+}$ reagents (including enantioselective variants)

- Chlorine-fluorine exchange reactions

- Direct fluorination using $\mathrm{F}_{2}$

In this contribution we will present a short overview of the first three reaction types.

\section{Deoxofluorination Reactions using Sulfur Tetrafluoride $\mathrm{SF}_{4}$}

$\mathrm{SF}_{4}$ has proved to be a particularly useful reagent for the selective fluorination of many oxygen-containing functional groups on laboratory scale. Of particular interest is the formation of trifluoromethyl groups, starting from carboxylic acids (Scheme 1), the preparation of difluorinated compounds using aldehydes and ketones as starting materials and of monofluorinated derivatives starting from alcohols (Scheme 2). Reactions employing $\mathrm{SF}_{4}$ require carefully tuned conditions, otherwise formation of tar-like or very impure product will result. Apart from the prevention of side reactions, the toxicity of the reagent along with the reaction conditions ranging from $-40{ }^{\circ} \mathrm{C}$ to 160 ${ }^{\circ} \mathrm{C}$ and pressures of up to 60 bar require rigorous safety measures. The combination of all necessary precautions and three decades of experience in $\mathrm{SF}_{4}$ chemistry ensures maximum safety and high success rate for custom-made $\mathrm{SF}_{4}$ fluorination at Solvias. These are carried out on up to a $500 \mathrm{~g}$ scale. The examples shown in the Schemes 1 and 2 are all 'real-world' preparations resulting in yields of at least 50\% (not optimized) and illustrate the broad range of substrates amenable to $\mathrm{SF}_{4}$ chemistry.

\section{Conversion of Anilines to Fluoro Aro- matics by the Modified Schiemann Reaction}

The Schiemann reaction is the most important method for introduction of fluorine atoms into aromatic rings. With the modified version, a broad variety of carbo- and heterocyclic primary amines can be converted to fluoro aromatics on both laboratory and technical scale. The diazotation step is usually performed with $\mathrm{NaNO}_{2}$ in pyridine-HF or anhydrous HF. HF serves as a solvent as well as a reagent. The dediazotation/fluorination is carried out by heating the reaction mixture above its decomposition temperature. In contrast to the traditional Baltz-Schiemann reaction where only gram amounts of isolated diazonium tetrafluoroborates can be thermally treated, the modified Schiemann reaction allows safe conversion of up to mole amounts of diazo compounds. A representative selec- 


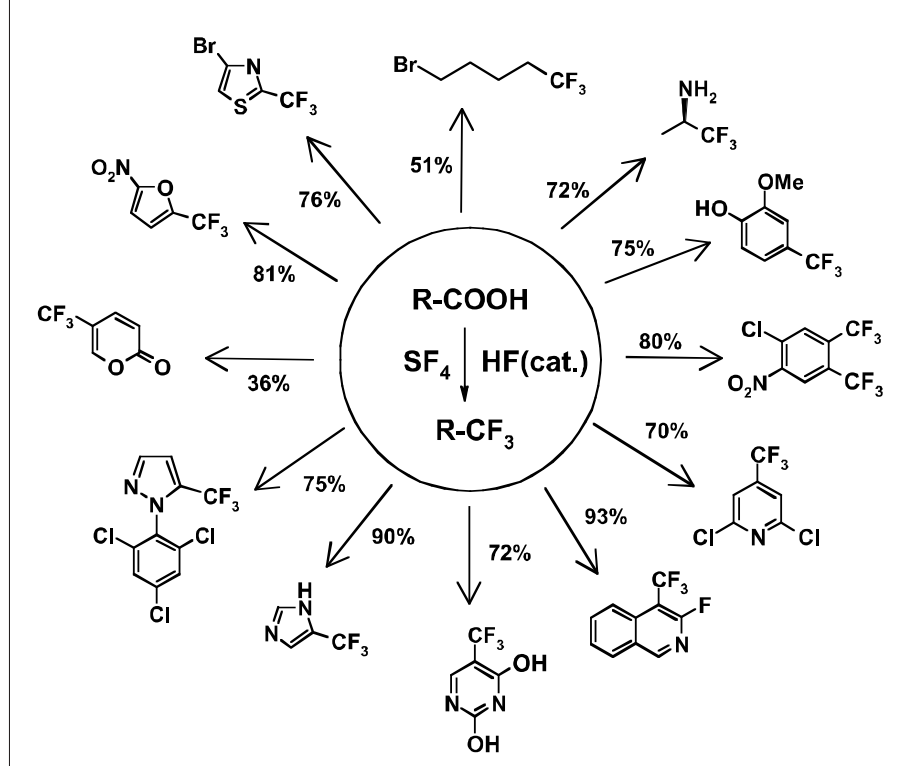

Scheme 1. Formation of $\mathrm{CF}_{3}$ groups from carboxylic acids with $\mathrm{SF}_{4}$ (yields not optimized)

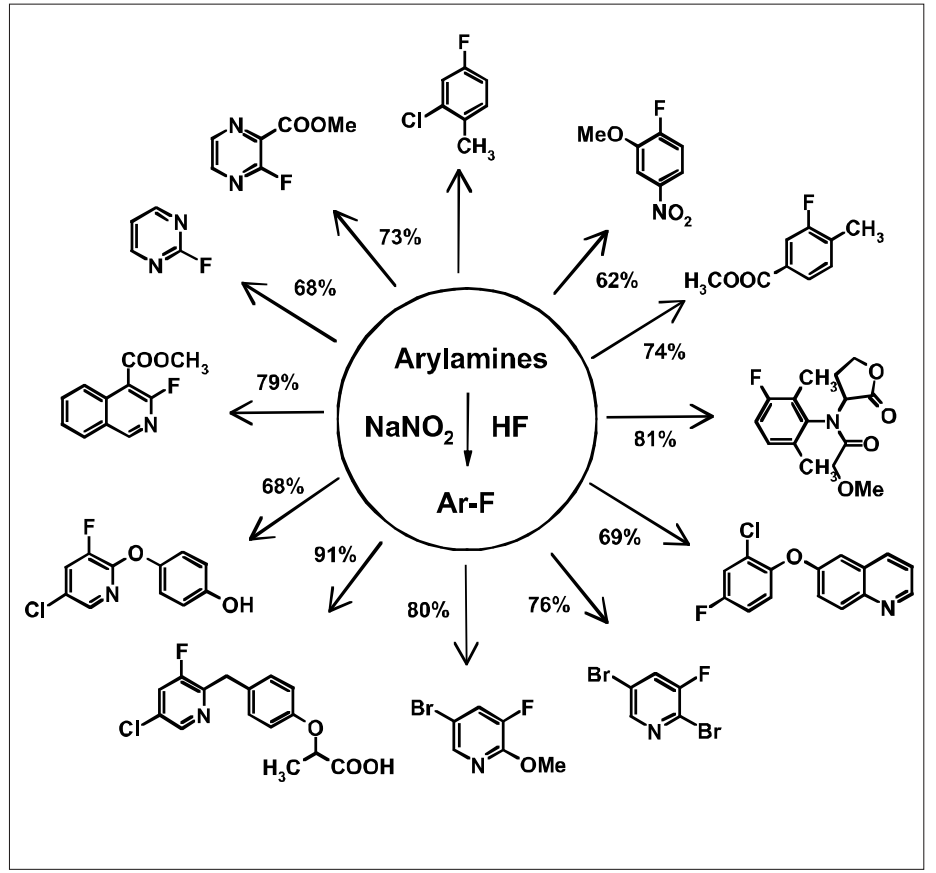

Scheme 3. Scope of the modified Schiemann reaction to prepare aromatic fluoro compounds (yields not optimized)

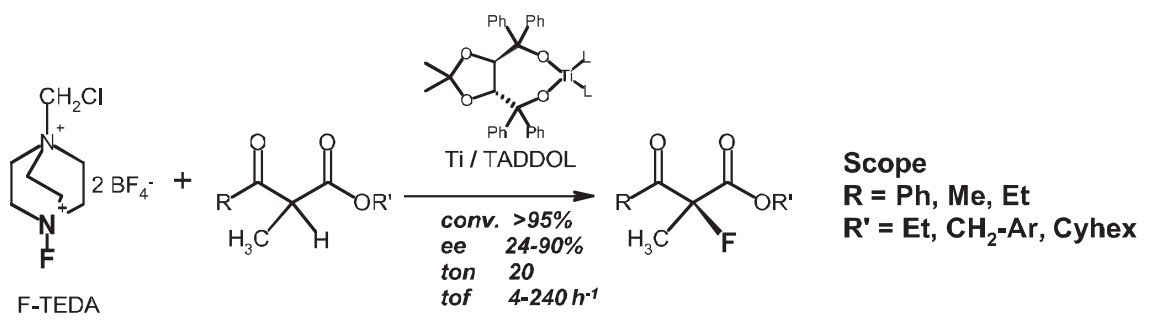

Scheme 4. Enantioselective catalytic fluorination of $\alpha$-substituted $\beta$-ketoesters

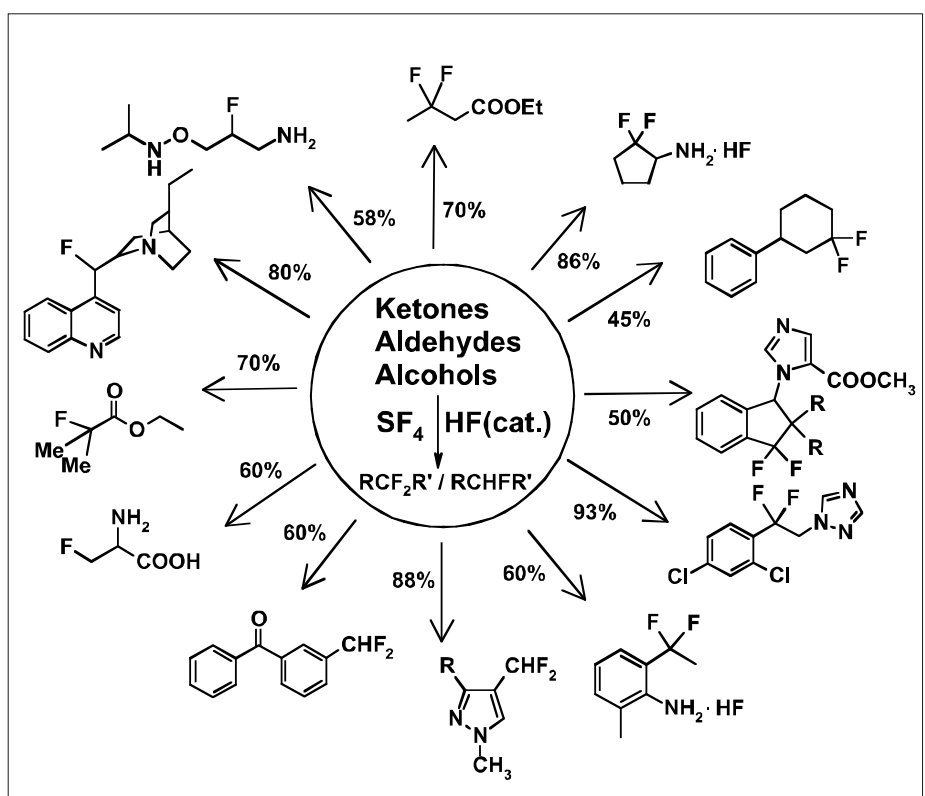

Scheme 2. Reaction products of alcohols, aldehydes and ketones with $\mathrm{SF}_{4}$ (yields not optimized) tion of preparative examples resulting in yields of at least 50\% (not optimized) is shown in Scheme 3.

\section{Enantioselective Catalytic Fluorination of $\beta$-Ketoesters}

While the application of various $\mathrm{F}^{+}$reagents is well known and widely practiced, we have recently developed the first enantioselective catalytic fluorination of $\beta$ ketoesters (Scheme 4) in collaboration with Prof. A. Togni of the ETH Zurich [2]. Very good conversions and medium to very good enantioselectivities can be obtained with a variety of $\alpha$-substituted $\beta$-ketoesters. With the same catalyst, selective chlorination and bromination is also possible.

\section{Outsourcing Preparative Fluorination?}

In contrast to chlorinated and brominated compounds, synthesis of fluorinated substances requires methods not readily applicable in standard organic laboratories. The reason lies in the toxicity and corrosiveness of many fluorination reagents. Therefore, specially equipped laboratories or even closed boxes with high-performance ventilation and gas washers and also special equipment such as plastic reactors or high-pressure autoclaves made of Monel $400^{\circledR}$ are often required. In addition, trained personnel with experience in handling toxic and aggressive chemicals, and high-pressure apparatus are a prerequisite as well.

This leads to the obvious question: If a fluorinated intermediate is not available commercially, do you prepare it yourself or outsource the preparation to a specialist? 


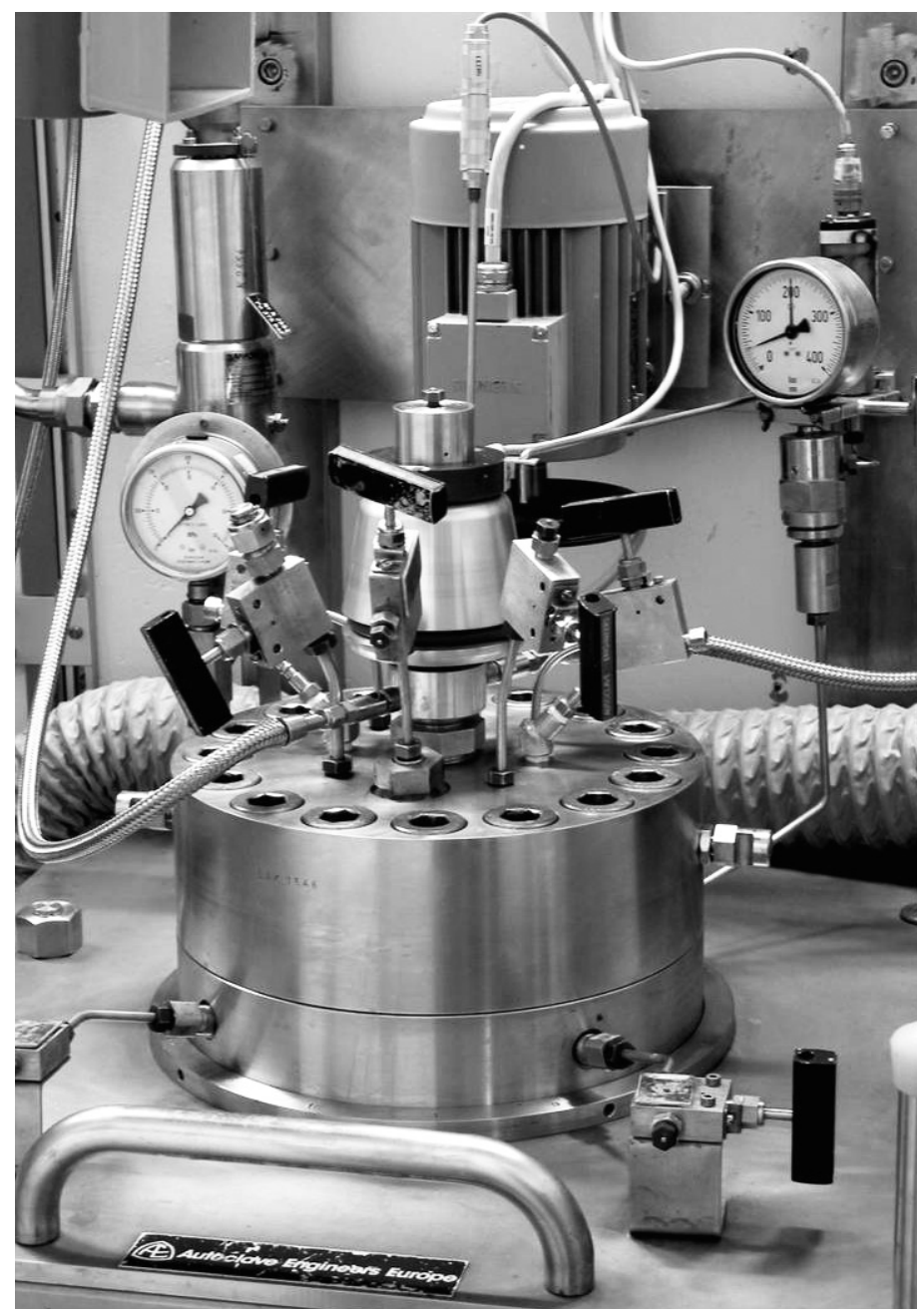

Fig. 16-litre autoclave for reactions between $-50^{\circ} \mathrm{C}$ up to $300{ }^{\circ} \mathrm{C}$ and up to 300 bar.

While many electrophilic fluorinations using $\mathrm{F}^{+}$-reagents such as F-TEDA (Scheme 4) can be carried out by experienced personnel on preparative scale without any special precautions, this is not recommended for reactions with reagents such as $\mathrm{HF}$ or $\mathrm{SF}_{4}$. For such preparations, outsourcing to a specialized company such as Solvias is clearly the method of choice.

For more than 30 years, all possible fluorination methods have been used at Solvias to prepare hundreds of fluorinated compounds on a preparative scale for our customers. Fluorinations are routinely carried out in various scales up to 16 litres. We not only have all equipment required (an example of a specialized autoclave is depicted in the Fig.), but also the know-how to successfully synthesize the target molecules on gram to kilogram scale and develop efficient processes for large-scale manufacturing. Besides the reactions described above, Solvias can also perform reactions with almost every fluorination reagent, including diluted $\mathrm{F}_{2}$ gas (e.g. $10 \% \mathrm{~F}_{2}$ in $\mathrm{N}_{2}$ ) under normal pressure and up to a $0.5 \mathrm{~mol}$ scale.

\section{Conclusions}

This brief overview demonstrates that several methods are available for the preparation of a variety of selectively fluorinated compounds on up to a kilogram scale. A serious issue associated with most fluorination chemistry is the toxic and often aggressive nature of most fluorination reagents. For this reason outsourcing small and larger scale preparation as well as process development to companies specialized in handling such reagents is highly recommended.

\section{Acknowledgements}

We thank Drs H.-U. Blaser and M. Thommen for helpful discussions.

Received: January 9, 2004

[1] Review: 'Methods of Organic Chemistry (Houben-Weyl)', Volume E 10a, Ed. B. Baasner, H. Hagemann, J.C. Tatlow, Thieme Verlag, Stuttgart, New York, 1999.

[2] a) L. Hintermann, A. Togni, Angew. Chem. 2000, 112, 530; b) L. Hintermann, A. Togni, Angew. Chem. Int. Ed. 2000, 39, 4359. 